\title{
Translation of neutrally evolving peptides provides a basis for de novo
}

gene evolution

Jorge Ruiz-Orera ${ }^{1,{ }^{*}}$, Pol Verdaguer-Grau ${ }^{2}$, José Luis Villanueva-Cañas ${ }^{1}$, Xavier Messeguer $^{2}$, M.Mar Albà ${ }^{1,3,{ }^{*}}$

${ }^{1}$ Evolutionary Genomics Group, Research Programme on Biomedical Informatics, Hospital del Mar Research Institute, Universitat Pompeu Fabra, Barcelona, Spain; ${ }^{2}$ Computer Sciences Department, Universitat Politècnica de Catalunya, Barcelona, Spain; ${ }^{3}$ Catalan Institution for Research and Advanced Studies, Barcelona, Spain.

*To whom correspondence should be addressed.

Running title: pervasive translation and de novo genes

Keywords: ribosome profiling, translation, de novo gene, long non-coding RNA, peptide, polymorphism, natural selection 


\section{Abstract}

2

3 There is accumulating evidence that some protein-coding genes have originated de novo from

4 previously non-coding genomic sequences. However, the processes underlying de novo gene

5 birth are still enigmatic. In particular, the appearance of a new functional protein seems highly

6 improbable unless there is already a pool of neutrally evolving peptides that are translated at

7 significant levels and that can at some point acquire new functions. Here we use deep ribosome

8 profiling sequencing data, together with proteomics and single nucleotide polymorphism

9 information, to search for these peptides. We find hundreds of open reading frames that are

10 translated, and that show no evolutionary conservation and no selective constraints. The data

11 suggests that the translation of these neutrally evolving peptides may be facilitated by the

12 chance occurrence of open reading frames with a favorable codon composition. We conclude

13 that the pervasive translation of the transcriptome provides plenty of material for the evolution of

14 new functional proteins. 
17 A large fraction of the genome is transcribed; this includes functional genes but also thousands

18 of transcripts that are not conserved across species and which show weak or no signatures of

19 natural selection ${ }^{1-3}$. Many of the latter transcripts are annotated as long non-coding RNAs

20 (IncRNAs) because they lack conserved long open reading frames (ORFs). Recent studies

21 based on the sequencing of ribosome-protected RNA fragments (ribosome profiling) have

22 reported that a surprisingly large number of these transcripts are associated with ribosomes and

23 may translate small proteins or peptides ${ }^{4-9}$. This is intriguing because only a small fraction of

24 them are likely to encode functional micropeptides ${ }^{10}$.

26 Each ribosome profiling experiment generates millions of ribosome footprints that are

27 subsequently mapped to the genome or the transcriptome to identify open reading frames

28 (ORFs) that are being translated ${ }^{11}$. The codon-by-codon movement of the ribosome along the

29 coding sequence results in a characteristic pattern of three nucleotide periodicity of the mapped

30 reads, which makes ribosome profiling a very useful method to detect novel events of

31 translation ${ }^{4,12,13}$. Given enough sequence coverage the technique can uncover low-abundant

32 small proteins that would be otherwise difficult to detect by standard proteomics

33 approaches ${ }^{14,15}$.

34

35 There is increasing evidence that some functional genes have originated de novo from 36 previously non-functional parts of the genome ${ }^{16-20}$. There are many protein-coding genes that 37 appear to be species-species; the encoded proteins are shorter than average and they tend to 38 evolve under more relaxed constraints than other genes ${ }^{21,22,20}$. There is experimental evidence 39 that at least some de novo genes are functional ${ }^{23,24}$. However, for a new protein to acquire a 40 function it first needs to be produced in the cell at significant amounts. Some of the new proteins 41 may be generated by the translation of randomly occurring ORFs in transcripts currently 42 annotated as non-coding ${ }^{7}$. As these proteins are not expected to be functional at first, they are 43 expected to evolve under no significant purifying selection, or neutrally. We previously showed 44 that, as a whole, putatively translated IncRNAs and young annotated protein-coding genes 
45 shared a number of similarities, such as small ORF size and weak selective constraints,

46 compared with more widely conserved genes ${ }^{8}$. This suggested that the number of proteins with

47 a recent evolutionary origin was larger than previously thought. However, it remained to be

48 determined if there existed a pool of young proteins that were translated at significant levels and

49 evolved in a neutral manner, as hypothesized for de novo gene evolution.

50

51 To assess the functional relevance of translated products one can use the ratio between the

52 number of non-synonymous and synonymous substitutions in the putative coding sequences ${ }^{4,5}$.

53 However, this method requires an alignment of at least two different homologous sequences. A

54 more general approach that can be used in the absence of homologues in other species is the

55 ratio between the number of non-synonymous and synonymous single nucleotide

56 polymorphisms, compared to the one expected under neutrality. Under no selection, non-

57 synonymous and synonymous polymorphisms persist at the same levels, whereas under

58 purifying selection there is a deficit of non-synonymous polymorphisms because some amino

59 acid changes disrupt the protein's function ${ }^{25}$. Single nucleotide polymorphism analysis can be

60 performed on a gene-by-gene basis or in pools of sequences that share certain features ${ }^{2,26}$.

61

62 Here we employ phylogenetic comparisons, ribosome profiling data and single nucleotide

63 polymorphism information to search for putative precursors of de novo genes in mouse. The

64 study renders visible a layer of protein expression that produces non-functional small proteins,

65 filling a gap in our understanding of the processes underlying de novo gene birth.

66

67 Results

68

69 Many ORFs outside annotated protein-coding sequences are translated

70

71 First we set out to identify translated open reading frames (ORFs) in mouse protein-coding

72 genes (codRNAs) and long non-coding RNAs (IncRNAs) using ribosome-profiling RNA-

73 sequencing (Ribo-Seq) data from eight different tissues and cell lines (Supplementary Table 1

74 and references therein). In contrast to RNA sequencing (RNA-Seq) reads, which are expected 
75 to cover the complete transcript, Ribo-Seq reads correspond to regions bound by ribosomes.

76 We mapped the RNA-Seq and Ribo-Seq reads to the mouse Ensembl gene annotations and,

77 for the sake of completeness, also to a set of previously obtained novel mouse transcripts that

78 did not correspond to annotated protein-coding genes or IncRNAs ${ }^{3}$.

79

80 We used the RibORF program ${ }^{4}$ to identify translated sequences among ORFs covered by at 81 least 10 Ribo-Seq reads in transcripts expressed in one or more tissues (Fig. 1a and

82 Supplementary Table 1). This program calculates a score for each ORF depending on the 3-

83 nucleotide periodicity and uniformity of the mapped reads. Using a highly stringent RibORF

84 score cut-off of $0.7^{4}$ we found that about $90 \%$ of the coding genes $(15,020)$, and $20 \%$ of the

85 annotated IncRNAs (539), were predicted to be translated in at least one sample. Additionally,

86 we identified 286 novel, non-annotated, expressed loci that also contained translated ORFs

87 (Fig. 1b). Annotated IncRNAs and novel transcripts mostly encoded small proteins (Fig. 1c) and

88 the two classes were merged.

89

90 Hundreds of translated ORFs have no homologues in other species and evolve neutrally

92 Putative precursors of de novo genes should be of recent origin and evolve under no purifying

93 selection. We performed exhaustive sequence similarity searches of the translated ORFs

94 against high coverage transcriptomes from human and rat as well as against the annotated

95 proteomes of 101 different eukaryotic species (Fig. 2a, Supplementary Table 2 for a list of

96 species, see Methods for more details). For these searches we discarded any peptides shorter

97 than 24 amino acids, as the detection of homologues may be compromised in such cases due

98 to lack of sufficient sequence information. We identified 1,980 translated ORFs that showed no

99 homology to expressed sequences in other species (class non-conserved or NC). This

100 represented about $10.4 \%$ of the translated ORFs.

101

102 Next, we measured the strength of selection acting on the putative proteins using a large

103 collection of mouse single nucleotide polymorphisms (SNPs) for the house mouse subspecies

104 Mus musculus castaneus ${ }^{27}$. We could map a total of 324,729 SNPs to the set of translated 
105 ORFs. We calculated the ratio between the number of observed non-synonymous and

106 synonymous SNPs (PN/PS(obs)) in conserved and non-conserved groups of ORFs, and

107 normalized it by the same ratio expected under neutrality (PN/PS(exp)). The expected PN/PS

108 was estimated using a table of nucleotide mutation frequencies in Mus musculus castaneus and

109 the observed codon frequencies in each set of sequences of interest (Supplementary Tables 3

110 to 5). This allowed us not only to compare the strength of selection across different sets of

111 sequences, as done in a previous study of ORFs translated from IncRNAs ${ }^{8}$, but also to discard

112 purifying selection if the normalized PN/PS was not significantly different from 1. Specifically, we

113 used a chi-square test that compared the number of observed and expected non-synonymous

114 and synonymous SNPs in each sequence set. The normalized PN/PS of conserved ORFs was

115 around 0.15 (Fig. $2 \mathrm{~b}$, p-value $<10^{-5}$ ), consistent with protein functionality. One example in this

116 group was Stannin ${ }^{28,29}$, a highly conserved peptide that regulates neuronal cell apoptosis (Fig.

$1173)$.

118

119 In the case of the non-conserved ORFs, the normalized PN/PS depended on the codon usage

120 bias or coding score (Supplementary Fig. 1 and 2). The coding score provides a measurement

121 of how similar the relative codon frequencies are to those observed in functional coding

122 sequences ${ }^{20,30,21}$. We divided the non-conserved ORFs in two groups: the first group, with high

123 coding scores $(\geq 0.1014, \mathrm{NC}-\mathrm{H})$, showed a weak but significant signature of selection (Fig. $2 \mathrm{~b}$,

124 p-value < 0.05); the second group, comprising the rest of ORFs, showed no significant deviation

125 from neutrality (NC-L)(see Fig. 3 for a specific example). The fist group is likely to contain some

126 species-specific functional genes, whereas the second group, the neutrally evolving ORFs,

127 represents new translated peptides with no function.

128

129 Neutrally evolving ORFs were found both in IncRNAs but also in many protein-coding genes

130 (Fig. 2b). In IncRNAs they were usually found in isolation or with other non-conserved ORFs,

131 whereas in coding genes they were in most cases located in alternative transcript isoforms of

132 genes already containing a conserved ORF. One possible way the secondary ORFs in protein-

133 coding genes could become new genes would be through gene duplication and inactivation of

134 the main ORF. The ORFs in IncRNAs and coding genes were merged into a single group, 
comprising 1,291 ORFs, for subsequent analyses (neutral ORFs).

\section{Validation of the set of neutrally evolving peptides}

139 The above analyses grouped the sequences into classes before computing the PN/PS ratio. In 140 general, ORF-by-ORF analysis was not possible because the ORFs were small and contained 141 too few SNPs. Nevertheless, 41 of the ORFs in the neutral set contained 10 or more SNPs, and 142 we decided to compute a normalized PN/PS ratio for these individual cases. The median PN/PS 143 of these ORFs was around 1 and the distribution of PN/PS values was very different from that 144 observed for conserved ORFs (Supplementary Fig. 3, Wilcoxon test, $p$-value $<10^{-5}$ ), consistent 145 with the previous results. Finally, we quantified the number of ORFs that contained SNPs that 146 generated premature stop codons, truncating more than half of the ORF, in the set of neutrally 147 evolving ORFs and in the set of conserved ORFs. In the first case we found 72 out of 1,291

148 ORFs that contained this type of mutation (5.6\%) and in the second case 296 out of 17,043 149 ORFs (1.74\%). Considering that neutral ORFs are in general much shorter than conserved 150 ORFs (median protein size 44 versus 412 amino acids), and thus less likely to accumulate 151 ORF-truncating mutations by chance alone, the data clearly indicates a strong excess of ORF152 truncating SNPs in neutral ORFs with respect to conserved ORFs.

154 We next calculated which was the percentage of neutral ORFs in the transcripts that were 155 detected as translated by our pipeline. This value was $30.5 \%(1,291$ out of 4,232$)$, about one 156 order of magnitude larger than the false positive rate estimated from two negative controls. The 157 first control was a set of ORFs derived from small nuclear and nucleolar RNAs (3.33\% false 158 positive rate, see example in Fig. 3), the second one was a set of ORFs in which the position of 159 the reads had been randomized (4.16\% false positive rate; see Methods for more details).

161 We used proteomics data from the PRIDE database ${ }^{31}$ to search for additional evidence of the 162 neutrally evolving peptides. Despite their small size (median 44 amino acids), a limiting factor 163 for their detection by standard proteomics-based techniques ${ }^{32}$, we found proteomics evidence 164 for 32 of the translated ORFs in this group. This represents $2.5 \%$ of the proteins in this set 
165 (compared to less than $0.2 \%$ false positive rate, see Methods). This fraction is similar to the one

166 obtained for conserved proteins subsampled to have a similar size distribution as the neutral

167 ORFs (2.9\%; in contrast, about $41 \%$ of all conserved ORFs have proteomics evidence). The

168 subset of ORFs with proteomics evidence did not deviate significantly from neutrality according

169 to the PN/PS-based test (Supplementary Table 5).

170

171 What determines that some ORFs, but not others, are translated?

172

173 Some transcripts contained relatively long ORFs but were not translated. One example of this

174 sort was the previously described de novo non-coding gene Poldi $i^{33}$ that lacked any evidence of

175 translation in the data we analyzed. We next asked which factors may influence the translation

176 of neutral ORFs. First, we inspected the translation initiation sequence context but did not

177 detect any clear differences between translated and non-translated neutral ORFs

178 (Supplementary Fig. 4). We then hypothesized that the ORF coding score could affect the

179 "translatability" of the transcript because codons that are abundant in coding sequences are

180 expected to be more efficiently translated than other codons ${ }^{34}$. Consistent with this hypothesis,

181 we found that the translated neutral ORFs exhibited higher coding scores than non-translated

182 ORFs (Fig. 4a, Translated versus non-translated Wilcoxon test, p-value $<10^{-5}$ ). Importantly, the

183 differences were maintained after subsampling the genes so that the two sets had the same

184 gene expression distribution (Fig. 4b, Wilcoxon test, p-value $<10^{-5}$ ). This is consistent with

185 codon composition having an effect per se in ORF translation. When controlling by coding

186 score, expression level, but not ORF length, was positively related to the detection of translation

187 (Fig. 4c).

188

189 It has been previously hypothesized that what distinguishes translated from non-translated

190 IncRNAs is the relative amount of the IncRNA in the cytoplasm with respect to the nucleus ${ }^{4}$.

191 However, we found evidence that some IncRNAs with nuclear functions, such as Malat1 and

192 Neat1, translated neutral ORFs, suggesting that the cytosolic fraction of a transcript can be

193 translated independently of its role or preferred location. Instead, we found that neutral ORFs

194 enriched in codons that are abundant in functional protein-coding genes could be translated 
195 more efficiently than other ORFs. This is consistent with the observation that abundant codons

196 enhance translation elongation ${ }^{35}$, whereas rare codons might affect the stability of the mRNA ${ }^{36}$.

197 These differences could result in compositional biases in newly emerged proteins.

\section{Discussion}

200

201 The molecular mechanisms underlying de novo gene evolution are still poorly understood ${ }^{17,18,37}$.

202 The sudden appearance of a new protein-coding gene from a genomic segment seems a priori

203 highly improbable, but the process becomes much more likely if the genome is already being

204 pervasively transcribed and translated outside functional protein-coding sequences. An excess

205 of transcription was already noted in the first large-scale cDNA sequencing efforts performed in

206 human and mouse ${ }^{38}$, and more recent studies have found a high rate of genome transcriptional

207 turnover when comparing closely related species ${ }^{39}$. Other works have found that many open

208 reading frames outside annotated protein-coding sequences can be translated ${ }^{4,5,7}$, raising the

209 possibility that they are an important source of de novo genes ${ }^{9,20,8}$.

210

211 The precursors of new functional proteins are expected to be translated at relatively high levels

212 and evolve under no purifying selection. Here we have detected hundreds of small proteins that

213 appear to be of recent evolutionary origin and that fulfill these properties. Their real number

214 could be much higher considering that many cell types and tissues have not yet been sampled.

215 According to recent estimations, the cost of transcription and translation in multicellular

216 organisms is probably too small to overcome genetic drift ${ }^{40}$. Therefore, if the peptides do not

217 affect the fitness per se, these activities may be effectively neutral. Our results are in line with

218 this prediction.

219

220 It has been proposed that nascent proteins are pre-adapted in the sense that only peptides that

221 do not have a harmful effect will be expressed at significant levels ${ }^{9}$, and that this may explain

222 the observed compositional differences between young proteins and intergenic sequences ${ }^{41}$.

223 Another possible source of bias is the codon composition of the sequence. According to our

224 results, ORFs that have a high coding score may be more efficiently translated than those that 
225 have a lower coding score. As a result, new proteins may have a more more coding-like

226 composition than the average random or intergenic sequence.

228 The process of de novo gene origination involves the gain of a useful function by a previously

229 non-functional sequence. The rate at which this happens remains to be determined but it has

230 been observed that many artificially generated peptides can function as secretion signals ${ }^{42}$, and

231 selection for ATP-binding activity in a library of randomly generated 80 amino acid polypeptides

232 successfully identified several candidates capable of binding to ATP $^{43}$. Recent experiments

233 performed in E.coli also suggest that random expressed sequences can often affect cellular

234 growth $^{44}$.

235

236 We have shown that the transcriptome is pervasively translated, and that this generates novel

237 peptides that evolve under no constraints. This set of translated peptides, and their sequences,

238 are expected to change rapidly during evolution, generating a myriad of opportunities for the

239 birth of new functional genes.

240

241 Methods

242

243 Transcript assembly

244

245 We used strand-specific polyA+RNA sequencing data (RNA-Seq) data from different mouse

246 and human tissues to assembly the species transcriptomes (Gene Expression Omnibus mouse

247 GSE69241 ${ }^{3}$, GSE43721 45 , and GSE43520 ${ }^{46}$; human GSE69241 ${ }^{3}$ ). The mouse RNA samples

248 were extracted from strain Balb/C. RNA-Seq reads were filtered by length ( $>25$ nucleotides)

249 and by quality using Condetri (v.2.2) ${ }^{47}$ with the following settings: $-\mathrm{hq}=30-\mathrm{lq}=10$. We aligned

250 the reads to the corresponding reference species genome with Tophat (v. 2.0.8, $-\mathrm{N} 3$, -a 5 and

$251-\mathrm{m} \mathrm{1})^{48}$. Multiple mapping to several locations in the genome was allowed unless otherwise

252 stated. We assembled the transcriptome with Stringtie $^{49}$, merging the reads from all the

253 samples, with parameters $-\mathrm{f} 0.01$, and $-\mathrm{M} 0.2$. We used the species transcriptome as a guide

254 (Ensembl v.75), including all annotated isoforms, but permitting the assembly of annotated and 
255 novel isoforms and genes (antisense, intergenic and intronic) as well. We excluded IncRNAs

256 that overlapped annotated pseudogenes or that showed significant sequence similarity to

257 known protein-coding sequences (BLASTP, e-value $<10^{-4}$ ). In the case of rat we employed a

258 previously generated transcript assembly ${ }^{50}$.

259

260 Ribosome profiling data

261

262 We used ribosome profiling data (Ribo-Seq) from 8 different mouse tissues or cell lines (see

263 Supplementary Table 1), obtained from Gene Expression Omnibus under accession numbers

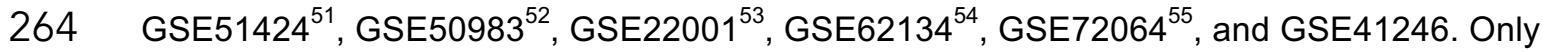

265 datasets corresponding to non-pathogenic conditions were considered. The reads from

266 experimental replicates were merged before using RibORF to increase the resolution of the

267 read periodicity, as done in the original RibORF paper ${ }^{4}$. For all analyses we considered only

268 genes expressed at significant levels in at least one sample (RNA-Seq fragments per kilobase

269 per Million mapped reads $($ FPKM $)>0.2)$. The expression of the genes detected in these

270 samples is expected to be highly representative of the Mus musculus species as a whole. We

271 mapped several brain RNA-Seq datasets from Mus musculus castaneus ${ }^{39}$ to the mouse

272 assembled transcriptome using NextGenMap ${ }^{56}$. As expected, the vast majority of the genes

273 expressed in brain samples from C57BL/6 mice ${ }^{51}$ also showed evidence of expression in Mus

274 musculus castaneus brain RNA samples ${ }^{39}$ (Supplementary Table 6).

275

276 We discarded anomalous reads (length $<26$ or $>33 \mathrm{nt}$ ) and reads that mapped to annotated

277 rRNAs and tRNAs in mouse from the Ribo-Seq sequencing datasets. Next, reads were mapped

278 to the assembled mouse genome (mm10) with Bowtie (v. 0.12.7, parameters -k $1-\mathrm{m} 20-\mathrm{n} 1--$

279 best --strata). Considering that the ORFs had to be extensively covered by reads to be

280 considered translated (high uniformity), we decided to include multiple mapped reads. This is

281 expected to increase the sensitivity to detect translation events, especially in gene families, but

282 also the number of false positives. We observed that using multiple mapped reads increased

283 the periodicity and uniformity values of a number of protein-coding genes, but that it had little

284 effect on translated ORFs from IncRNAs (Supplementary Fig. 5). We used the mapping of the 
285 Ribo-Seq reads to the complete set of annotated coding sequences in mouse to compute the 286 position of the P-site (second binding site for tRNA in the ribosome) for reads of different size, 287 as previously described ${ }^{11,13}$.

Identification of translated ORFs

290

291 We predicted all translated ORFs (ATG to STOP) with a minimum length of 9 amino acids in the 292 transcripts with RibORF (v.0.1 $)^{4}$. Only ORFs with a minimum of 10 mapped Ribo-Seq reads 293 were considered. The RibORF classifier is based on a support vector machine algorithm, 294 originally applied to human transcripts. The input parameters are the 3-nucleotide periodicity 295 and the uniformity of the reads. The periodicity refers to the distribution of the reads in the three 296 different possible frames; two values are computed, $\mathrm{f} 1$, which is the fraction of reads that 297 correspond to the correct frame, and $\mathrm{f} 2$, which is the fraction of reads in position +1 of the 298 correct frame. The uniformity corresponds to the percentage of maximum entropy, a value of 1 299 indicates a completely even distribution of reads across regions. For each ORF the program 300 computes a score that depends on the periodicity and the uniformity ${ }^{4}$. We used the same score 301 cut-off as in the original paper ( $\geq 0.7$ ), which had a reported false positive rate of $0.67 \%$ and a 302 false negative rate of $2.5 \%$.

303

304 We eliminated any redundancy in the translated ORFs by taking the longest ORF when several 305 overlapping translated ORFs were detected in the same gene. The identification of translated

306 ORFs was done separately for the different tissues (Supplementary Table 1). Differences in the number of translated ORFs in different tissues were related to the depth of sequencing and the number of reads that mapped to the most highly expressed proteins (Supplementary Fig. 6 and 7 , respectively). When translation of the same ORF was detected in several tissues, we kept the 310 information for the tissue with the highest RibORF score.

\section{Sequence conservation}

314 We searched for mouse ORF homologues in the human and rat transcriptomes using TBLASTN 
315 (limited to one strand, e-value $\left.<10^{-4}\right)^{57}$. We also performed sequence similarity searches 316 against the annotated proteomes of 67 mammalian-species and 34 non-mammalian eukaryotes

317 from a diverse range of groups compiled in a previous study ${ }^{50}$, using BLASTP (e-value $<10^{-4}$ ).

318 For these searches we only considered query proteins of size 24 amino acids or longer, as

319 shorter proteins may not contain sufficient information to perform homology searches. Mouse

320 ORFs that did not have any homology hits in other species were classified as non-conserved,

321 the rest as conserved. Non-conserved ORFs located upstream or downstream of another longer

322 ORF in a conserved transcript (UORFs and dORFs) were excluded from this analysis.

324 We inspected the rat genomic syntenic regions of translated ORFs using LiftOver ${ }^{58}$. We

325 classified the ORFs in two groups depending on whether the ORF was truncated in rat or not

326 (the truncation had to affect more than half of the protein). For neutrally evolving ORFs the

327 number of cases in which the ORF was truncated was similar to the number of cases in which it

328 was not truncated, and in both cases the polymorphism patterns were consistent with neutrality

329 (Supplementary Table 5). This indicated that, for this group, the presence of a similar ORF in rat 330 does not imply functional conservation of the ORF. Therefore, we did not use information on rat 331 genomic synteny to classify the genes as conserved/non-conserved.

\section{Coding score}

335 We used a previously described metric based on hexamer frequencies to calculate the coding 336 score of the sequences ${ }^{8}$. The method uses a table of pre-calculated hexamer scores that 337 measure the relative frequency of each hexamer in coding versus non-coding sequences.

338 These scores are then used to evaluate the coding propensity of a sequence based on its 339 hexamer composition. The method has been implemented in a computational program called

340 CIPHER that can be used to predict putative translated ORFs and can be accessed online

341 (http://evolutionarygenomics.upf.edu/cipher).

343 Comparison of the coding score in translated and non-translated ORFs 
345 For comparative purposes we generated a set of non-translated ORFs that was completely

346 independent from the translated ORFs (Fig. 4). We selected expressed loci that did not contain

347 any translated ORF and selected the longest predicted ORF sequence. An ORF was

348 considered not to be translated if it had less than 10 Ribo-Seq mapped reads, or a RibORF

349 score $<0.7$. Selecting the longest ORF was justified by the fact that, in translated ORFs, the

350 ORF with the highest number of mapped Ribo-Seq reads was usually the longest ORF $(75.7 \%$

351 for codRNAs and $84 \%$ for IncRNAs). We also generated a set of 4,013 randomly taken ORFs

352 from introns, after discarding ORFs that showed significant sequence similarity to known

353 proteins from the same species (BLASTP, e-value $<10^{-4}$ ).

354

355 In order to investigate the influence of gene expression level in our capacity to detect translation

356 of an ORF we subsampled the set of translated neutral ORFs and the set of non-translated

357 ORFs so that the two sets had equivalent gene expression value distributions. As gene

358 expression units we used Fragments Per Kilobase per Million mapped reads (FPKM), and we

359 selected the highest FPKM value across the different tissues examined. We applied the same

360 procedure to control for coding score values when evaluating the effect of gene expression level

361 and ORF length.

362

363 Ribosome profiling controls

364

365 We inspected the ribosome profiling patterns of neutral ORFs with respect to the rest of

366 translated ORFs ("functional"). The number of Ribo-Seq reads per base was in general lower in

367 neutral ORFs than in functional ORFs, although there was a significant overlap in the two

368 distributions (Supplementary Fig. 8). Translated sequences were characterized by strong 3-

369 nucleotide periodicity and this property showed a significant correlation across pairs of tissues,

370 both for neutral and functional ORFs (Supplementary Fig. 8 and 9). After subsampling the

371 functional ORFs to match the neutral ORFs with regards to ORF size and number of Ribo-Seq

372 reads, the two sets showed similar RibORF score, periodicity and uniformity value distributions

373 (Supplementary Fig. 10).

374 
375 We generated a negative control set by taking randomly occurring ORFs in mouse small

376 nuclear and nucleolar RNAs ("sRNAs"). These ORFs were required to have at least 10 Ribo-

377 Seq mapped reads and were processed in the same manner as the main set of ORFs under

378 study. Only 10 out of 304 ORFs in "sRNA" had a RibORF score equal or higher than 0.7 (3.33\%

379 false positive rate). This control was characterized by low uniformity and low read periodicity

380 (Supplementary Fig. 8). A second negative control in which all parameters were equal to those

381 observed in neutral ORFs, but the reads were randomly distributed in the three frames, resulted

382 in 176 out of 4,232 ORFs classified as translated ("Random", $4.16 \%$ false positive rate).

383

384 We used a similar strategy to estimate the fraction of translated non-conserved ORFs (NC) over 385 all non-conserved ORF and the fraction of translated neutral ORFs over all neutral ORFs (NC-

386 L). Non-translated ORFs were those covered by 10 or more Ribo-Seq reads but with a RibORF 387 score lower than 0.7 . We followed the same procedure described for the translated ORFs

388 (Figure 2a) to classify them into classes. We detected translation for $33.1 \%$ of the non-

389 conserved ORFs $(1,980$ out of 5,975$)$ and $30.5 \%$ of the neutral ORFs $(1,291$ out of 4,232$)$.

390 These values are much higher than the estimated false positive rate.

392 We also generated a positive control set composed of 2,163 randomly taken annotated mouse 393 coding sequences with protein evidence in Uniprot. With this control we estimated a false 394 negative rate of $2.54 \%$.

395

396 Single nucleotide polymorphism analysis

398 We obtained single nucleotide polymorphism (SNP) data from 20 individuals of the house 399 mouse subspecies Mus musculus castaneus ${ }^{27}$. We classified SNPs in ORFs as non400 synonymous (PN, amino acid altering) and synonymous (PS, not amino-acid altering). We 401 calculated the PN/PS ratio in each ORF group by using the sum of PN and PS in all the 402 sequences ((PN/PS)obs). We calculated the expected PN/PS under neutrality ((PN/PS)exp) 403 using the mutation frequencies between pairs of nucleotides in Mus musculus castaneus and 404 the codon composition of the different sequences or sets of sequences under study 
405 (Supplementary Tables 3 and 4). The observed transition to transversion ratio was 4.42, very 406 similar to the 4.26 value obtained in early observations based on mouse-rat divergence data ${ }^{59}$.

407 We tested for purifying selection using a chi-square test with one degree of freedom

408 (Supplementary Table 5). Positively selected mutations are rapidly fixed in the population and 409 their effect is expected to be negligible when using SNP data.

410

\section{$411 \quad$ Proteomics data}

412

413 We used the proteomics database PRIDE ${ }^{31}$ to search for peptide matches in the proteins 414 encoded by various gene sets. For a protein to have proteomics evidence, we required at least 415 two distinct perfect matches of peptides that did not map to any other protein in the dataset, 416 allowing for up to two mismatches. Under these conditions the false positive rate was estimated 417 to be below $0.2 \%$ in a previous study ${ }^{50}$. The estimation was based on the results obtained for 418 ORFs in which the amino acid sequence was randomized and for ORFs in intronic sequences.

$420 \quad$ Statistical tests and plots

422 The generation of plots and statistical tests was performed with the R package ${ }^{60}$.

423

424 Code availability

425

426 Code for calculating the coding score is available at https://github.com/jorruior/CIPHER and for 427 calculating the expected PN/PS at https://figshare.com/articles/computePNPS c/5085706.

\section{Data availability}

431 The datasets generated during the current study are available from figshare repository, 432 http://dx.doi.org/10.6084/m9.figshare.4702375.

\section{$434 \quad$ Figure legends}


436 Figure 1. Detection of translated ORFs. a. Workflow to identify translated ORFs. Ribosome 437 profiling (Ribo-Seq) reads, corresponding to ribosome-protected fragments, are mapped to all 438 predicted canonical ORFs with length $\geq 30$ nucleotides in transcripts. This is performed with 439 single-nucleotide resolution after computing the read $\mathrm{P}$-site per each read length. In each ORF, 440 reads per frame and read uniformity are evaluated by RiboORF. b-c. Number of translated and 441 non-translated expressed loci (b) and ORFs (c) using a RibORF cut-off of 0.7. The original data 442 come from eight different mouse tissues (Supplementary Table 1). The translated ORFs have 443 been divided into small ORFs (smORF, $<100$ aa) and long ORFs ( $\geq 100$ aa), depending on 444 their length. "Novel" corresponds to expressed loci that do not correspond to annotated protein445 coding genes or IncRNAs, they are merged with IncRNAs as they mostly contain short non446 conserved ORFs.

448 Figure 2. Identification of selection signatures. a. Workflow to identify conserved and non449 conserved ORFs. Translated ORFs shorter than 24 amino acids, as well as non-conserved 450 upstream and downstream ORF in conserved transcripts (uORFs and dORFs, see Methods), 451 were filtered out. Any ORF with at least one BLAST match in another species was classified as 452 conserved (C), otherwise it was classified as non-conserved (NC). Non-conserved ORFs with a 453 high coding score value $(\geq 0.1014)$ were classified as NC-H, and the rest were classified as NC454 L. b. Analysis of selective constraints in translated ORFs. PN/PS (obs/exp) refers to the 455 normalized ratio between non-synonymous (PN) and synonymous (PS) single nucleotide 456 polymorphisms; a value of 1 is expected in the absence of selection at the protein level.

457 Conserved and NC-H ORFs showed significant purifying selection signatures. In contrast, NC-L 458 ORFs did not show evidence of purifying selection at the protein level. Some conserved ORFs 459 in IncRNAs are likely to encode functional micropeptides. Differences between observed and 460 expected PN/PS were assessed with a chi-square test, ${ }^{*} p$ - value $<0.05$, ${ }^{* * *} p$-value $<10^{-5}$. 461 Error bars indicate the standard error of the sample proportion. Numbers of ORFs for the 462 different categories are also displayed.

463

464 Figure 3. Three nucleotide periodicity of translated ORFs. The mapping of Ribo-Seq reads 
465 on different types of ORFs is shown. The $Y$ axis represents the log-number of reads, the $X$ axis 466 the positions in the ORF. The reads show strong frame bias in the functional (conserved) and 467 the neutral (NC-L) examples, with a preponderance of in-frame reads (green) versus off-frame 468 reads (red and blue), while there is no consistent frame bias in the negative control (SNORA18).

469 The exon/intron structure and the amino acid sequence for translated ORFs is also shown.

470

471 Figure 4. Factors influencing the translation of neutrally evolving ORFs. a. Influence of 472 coding score in the translatability of neutrally evolving ORFs. Translated ORFs showed 473 significantly higher coding score than non-translated ORFs, both sets had significantly higher 474 coding scores than introns (Wilcoxon test $p$-value $<10^{-5}$, indicated by ${ }^{* * *}$ ). b. Influence of coding 475 score in the translatability of ORFs after controlling for gene expression values. Translated 476 ORFs showed significantly higher coding score values than non-translated ORFs (Wilcoxon test

$477 \mathrm{p}$-value $<10^{-5}$ ). c. Influence of gene expression level and ORF length in the translatability of 478 neutral ORFs after controlling for coding score. Translated ORFs showed significantly higher 479 FPKM values than non-translated ORFs (Wilcoxon test $p$-value $<10^{-5}$ ); differences in length 480 were not significant.

\section{References}

481 1. Kutter, C. et al. Rapid turnover of long noncoding RNAs and the evolution of gene $482 \quad$ expression. PLoS Genet. 8, e1002841 (2012).

483 2. Wiberg, R. A. W. et al. Assessing Recent Selection and Functionality at Long Noncoding 484 RNA Loci in the Mouse Genome. Genome Biol. Evol. 7, 2432-44 (2015).

485 3. Ruiz-Orera, J. et al. Origins of De Novo Genes in Human and Chimpanzee. PLOS $486 \quad$ Genet. 11, e1005721 (2015).

487 4. Ji, Z., Song, R., Regev, A. \& Struhl, K. Many IncRNAs, 5’UTRs, and pseudogenes are 488 translated and some are likely to express functional proteins. Elife 4, e08890 (2015).

489 5. Raj, A. et al. Thousands of novel translated open reading frames in humans inferred by 490 ribosome footprint profiling. Elife 5, e13328 (2016).

491 6. Ingolia, N. T., Lareau, L. F. \& Weissman, J. S. Ribosome profiling of mouse embryonic 
stem cells reveals the complexity and dynamics of mammalian proteomes. Cell 147,

$493 \quad 789-802(2011)$.

494 7. Ingolia, N. T. et al. Ribosome Profiling Reveals Pervasive Translation Outside of 495 Annotated Protein-Coding Genes. Cell Rep. 8, 1365-79 (2014).

496 8. Ruiz-Orera, J., Messeguer, X., Subirana, J. A. \& Alba, M. M. Long non-coding RNAs as a source of new peptides. Elife 3, e03523 (2014).

498 9. Wilson, B. A. \& Masel, J. Putatively noncoding transcripts show extensive association with ribosomes. Genome Biol. Evol. 3, 1245-52 (2011).

500 10. Couso, J.-P. \& Patraquim, P. Classification and function of small open reading frames. Nat. Rev. Mol. Cell Biol. 18, 575-589 (2017).

502 11. Ingolia, N. T., Ghaemmaghami, S., Newman, J. R. S. \& Weissman, J. S. Genome-wide 503 analysis in vivo of translation with nucleotide resolution using ribosome profiling. Science 324, 218-23 (2009).

505

Bazzini, A. A. et al. Identification of small ORFs in vertebrates using ribosome footprinting and evolutionary conservation. EMBO J. 33, 981-93 (2014).

13. Calviello, L. et al. Detecting actively translated open reading frames in ribosome profiling data. Nat Meth 13, 165-170 (2016).

509

14. Aspden, J. L. et al. Extensive translation of small ORFs revealed by Poly-Ribo-Seq. Elife e03528 (2014).

15. Mackowiak, S. D. et al. Extensive identification and analysis of conserved small ORFs in animals. Genome Biol. 16, 1-21 (2015).

513 16. Begun, D. J., Lindfors, H. A., Kern, A. D. \& Jones, C. D. Evidence for de Novo Evolution 514 of Testis-Expressed Genes in the Drosophila yakuba/Drosophila erecta Clade. Genetics 176, 1131-1137 (2006).

516 17. Tautz, D. \& Domazet-Lošo, T. The evolutionary origin of orphan genes. Nat. Rev. Genet. $517 \quad$ 12, 692-702 (2011).

518 18. McLysaght, A. \& Hurst, L. D. Open questions in the study of de novo genes: what, how and why. Nat. Rev. Genet. 17, 567-578 (2016).

520 19. Zhao, L., Saelao, P., Jones, C. D. \& Begun, D. J. Origin and spread of de novo genes in Drosophila melanogaster populations. Science 343, 769-72 (2014). 
522 20. Carvunis, A.-R. et al. Proto-genes and de novo gene birth. Nature 487, 370-4 (2012).

523 21. Toll-Riera, M. et al. Origin of primate orphan genes: a comparative genomics approach.

524 Mol. Biol. Evol. 26, 603-12 (2009).

525 22. Cai, J. J. \& Petrov, D. A. Relaxed purifying selection and possibly high rate of adaptation 526 in primate lineage-specific genes. Genome Biol. Evol. 2, 393-409 (2010).

527 23. Chen, S., Zhang, Y. E. \& Long, M. New Genes in Drosophila Quickly Become Essential. 528 Science (80-. ). 330, 1682-1685 (2010).

529 24. Reinhardt, J. A. et al. De novo ORFs in Drosophila are important to organismal fitness 530 and evolved rapidly from previously non-coding sequences. PLoS Genet. 9, e1003860 $531 \quad$ (2013).

532 25. Sunyaev, S., Kondrashov, F. A., Bork, P. \& Ramensky, V. Impact of selection, mutation rate and genetic drift on human genetic variation. Hum. Mol. Genet. 12, 3325-3330

534 (2003).

535 26. Gayà-Vidal, M. \& Albà, M. M. Uncovering adaptive evolution in the human lineage. BMC Genomics 15, 599 (2014).

537 27. Harr, B. et al. Genomic resources for wild populations of the house mouse, Mus musculus and its close relative Mus spretus. Sci. Data 3, 160075 (2016).

28. Buck-Koehntop, B. A., Mascioni, A., Buffy, J. J. \& Veglia, G. Structure, dynamics, and membrane topology of stannin: a mediator of neuronal cell apoptosis induced by trimethyltin chloride. J. Mol. Biol. 354, 652-65 (2005).

29. Pueyo, J. I. et al. Hemotin, a Regulator of Phagocytosis Encoded by a Small ORF and Conserved across Metazoans. PLoS Biol 14, e1002395 (2016).

30. Plotkin, J. B. \& Kudla, G. Synonymous but not the same: the causes and consequences of codon bias. Nat. Rev. Genet. (2011). doi:10.1038/nrg2899

31. Vizcaíno, J. A. et al. 2016 update of the PRIDE database and its related tools. Nucleic Acids Res. 44, D447-D456 (2016).

32. Slavoff, S. A. et al. Peptidomic discovery of short open reading frame-encoded peptides in human cells. Nat. Chem. Biol. 9, 59-64 (2013). an intergenic region. Curr. Biol. 19, 1527-31 (2009). 
552 34. Dana, A. \& Tuller, T. The effect of tRNA levels on decoding times of mRNA codons.

$553 \quad$ Nucleic Acids Res. 42, 9171-81 (2014).

554 35. Yu, C. et al. Codon Usage Influences the Local Rate of Translation Elongation to 555 Regulate Co-translational Protein Folding. Mol. Cell 59, 744-754 (2015).

556 36. Presnyak, V. et al. Codon optimality is a major determinant of mRNA stability. Cell 160, $557 \quad 1111-24(2015)$.

558 37. Schlötterer, C. Genes from scratch - the evolutionary fate of de novo genes. Trends $559 \quad$ Genet. (2015).

560 38. Okazaki, Y. et al. Analysis of the mouse transcriptome based on functional annotation of 60,770 full-length cDNAs. Nature 420, 563-73 (2002).

562 39. Neme, R. \& Tautz, D. Fast turnover of genome transcription across evolutionary time exposes entire non-coding DNA to de novo gene emergence. Elife 5, e09977 (2016).

564 40. Lynch, M. \& Marinov, G. K. The bioenergetic costs of a gene. Proc. Natl. Acad. Sci. U. S. A. 112, 15690-5 (2015).

566 41. Wilson, B. A., Foy, S. G., Neme, R. \& Masel, J. Young Genes are Highly Disordered as Predicted by the Preadaptation Hypothesis of De Novo Gene Birth. Nat. Ecol. Evol. 1, 146 (2017). replace the secretion signal sequence of yeast invertase. Science $\mathbf{2 3 5}, \mathbf{3 1 2 - 7}(1987)$. Nature 410, 715-718 (2001).

573 44. Neme, R., Amador, C., Yildirim, B., McConnell, E. \& Tautz, D. Random sequences are 574 an abundant source of bioactive RNAs or peptides. Nat. Ecol. Evol. 1, 217 (2017).

575 45. Soumillon, M. et al. Cellular source and mechanisms of high transcriptome complexity in 576 the mammalian testis. Cell Rep. 3, 2179-90 (2013).

577 46. Necsulea, A. et al. The evolution of IncRNA repertoires and expression patterns in 578 tetrapods. Nature 505, 635-40 (2014).

579 47. Smeds, L. \& Künstner, A. ConDeTri--a content dependent read trimmer for Illumina data. $580 \quad$ PLoS One 6, e26314 (2011).

581 48. Kim, D. et al. TopHat2: accurate alignment of transcriptomes in the presence of 
insertions, deletions and gene fusions. Genome Biol. 14, R36 (2013).

49. Pertea, M. et al. StringTie enables improved reconstruction of a transcriptome from RNA-seq reads. Nat Biotech 33, 290-295 (2015).

50. Luis Villanueva-Cañas, J. et al. New Genes and Functional Innovation in Mammals. Genome Biol. Evol. 9, 1886-1900 (2017).

51. Gonzalez, C. et al. Ribosome profiling reveals a cell-type-specific translational landscape in brain tumors. J. Neurosci. 34, 10924-36 (2014).

52. Castañeda, J. et al. Reduced pachytene piRNAs and translation underlie spermiogenic

53. Guo, H., Ingolia, N. T., Weissman, J. S. \& Bartel, D. P. Mammalian microRNAs predominantly act to decrease target mRNA levels. Nature 466, 835-40 (2010).

54. Diaz-Munoz, M. D. et al. The RNA-binding protein HuR is essential for the B cell antibody response. Nat Immunol 16, 415-425 (2015).

55. Cho, J. et al. Multiple repressive mechanisms in the hippocampus during memory formation. Science 350, 82-87 (2015).

56. Sedlazeck, F. J., Rescheneder, P. \& von Haeseler, A. NextGenMap: fast and accurate read mapping in highly polymorphic genomes. Bioinformatics 29, 2790-2791 (2013).

57. Altschul, S. F. et al. Gapped BLAST and PSI-BLAST: a new generation of protein database search programs. Nucleic Acids Res. 25, 3389-402 (1997).

58. Karolchik, D. et al. The UCSC Genome Browser database: 2014 update. Nucleic Acids Res. 42, D764-D770 (2014).

59. Rosenberg, M. S., Subramanian, S. \& Kumar, S. Patterns of Transitional Mutation Biases Within and Among Mammalian Genomes. Mol. Biol. Evol. 20, 988-993 (2003).

60. R Development Core Team. R: a language and environment for statistical computing. $R$ Foundation for Statistical Computing, Vienna, Austria (2016).

\section{Acknowledgements}

609

610 We are grateful for valuable discussions with many colleagues during this study. The work was 611 funded by grants BFU2012-36820, BFU2015-65235-P and TIN2015-69175-C4-3-R from 
612 Ministerio de Economía e Innovación (Spanish Government) and co-funded by FEDER (EC).

613 We also received funding from Agència de Gestió d'Ajuts Universitaris i de Recerca Generalitat

614 de Catalunya (AGAUR), grant number 2014SGR1121.

615

\section{Author contributions}

617 J.R-O. and M.M.A. conceived the study, interpreted the data and wrote the paper. J.R-O.

618 performed most of the analyses including the transcript assemblies, identification of translated

619 open reading frames, BLAST searches, SNP mapping and generation of controls. J.R-O., P.V-

620 G. and J.L.V-C wrote code and performed analyses on the coding score. X.M. wrote code to

621 calculate the expected SNP frequencies. M.M.A. coordinated the study.

622

623 Competing interests

624 The authors declare no competing financial interests.

625 


\section{Figure 1}

a

Polysomes

Ribosome

footprinting

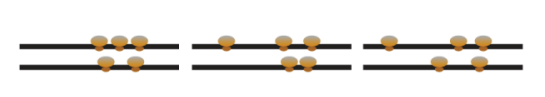

ORF prediction

$$
2 \approx 2-2202-2
$$

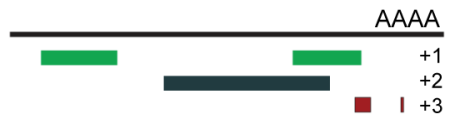

Compute P-site

Map reads

to positions

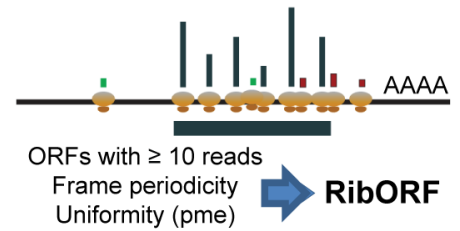

b

Number of genes

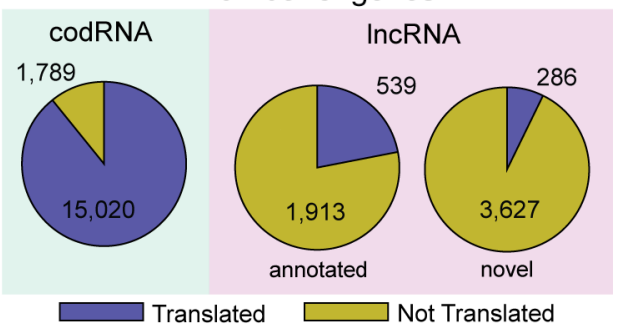

C

Number of ORFs

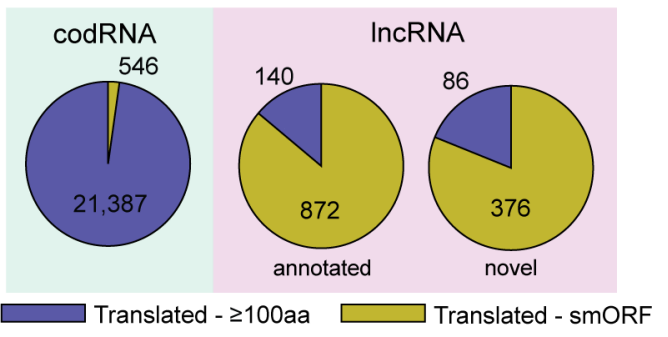

628 


\section{Figure 2}

a

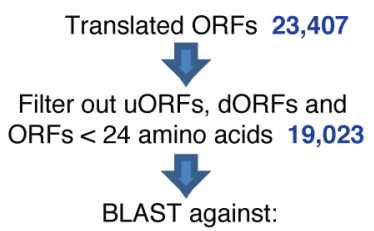

a) human and rat transcriptomes

b) annotated proteomes for 101 species

1

Conserved (C) Non-conserved (NC) 17,043 Compute coding score (CS

$\geq 0.1014$

Non-conserved - Non-conserved High score (NC-H) Low score (NC-L) 689 b

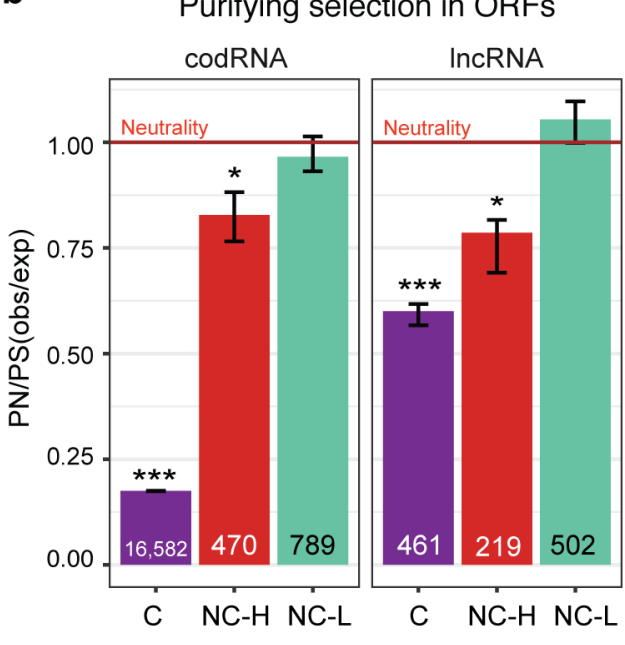


632 Figure 3

Stannin (Functional)

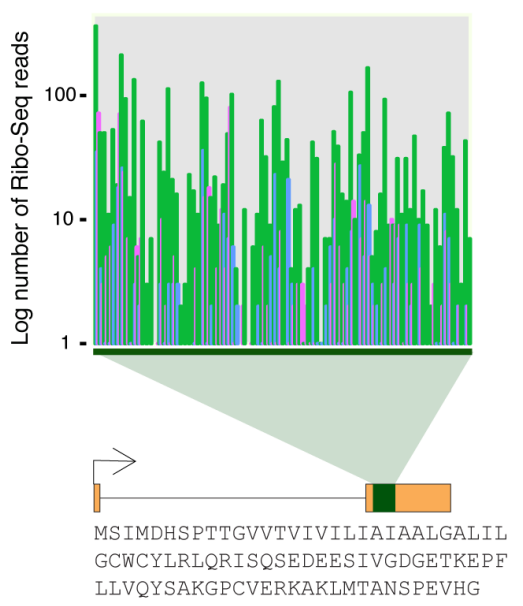

XLOC_007614 (Neutral)

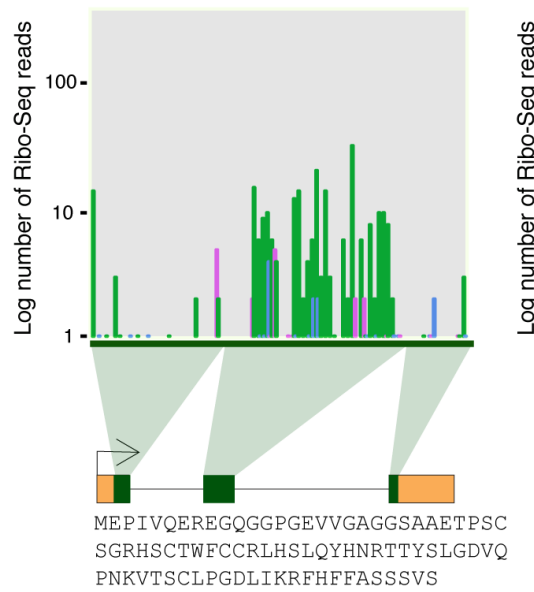

PNKVTSCLPGDLIKRFHFFASSSVS
SNORA18 (snoRNA)

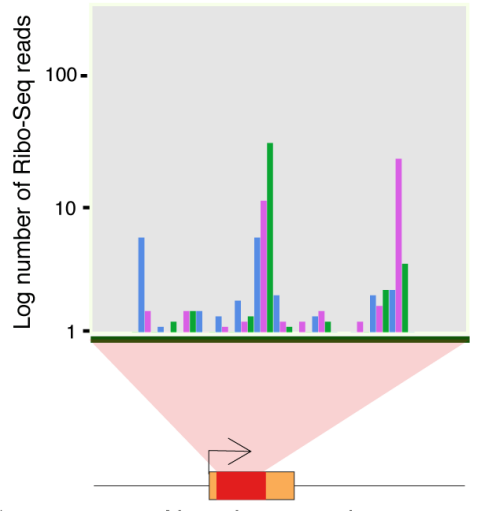

Negative control

633

634 


\section{Figure 4}

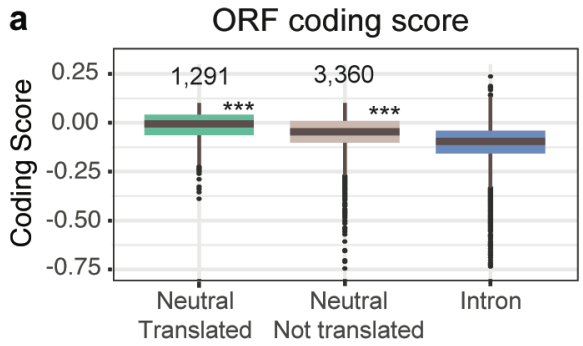

C
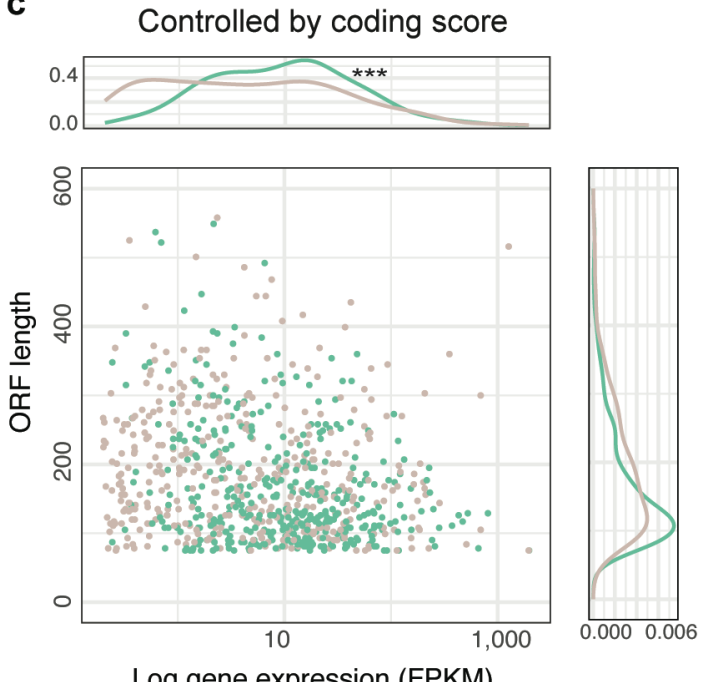

b Controlled by gene expresion

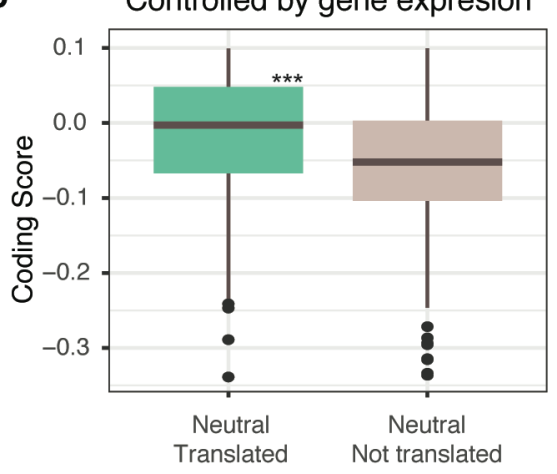

og gene expression (FPKM)

Neutral - Translated

Neutral - Not translated 